\title{
Fatigue damage monitoring in polymeric composites using multiple fiber bragg gratings
}

\author{
C.S. Shin *, C.C. Chiang \\ Department of Mechanical Engineering, National Taiwan University, No.1, Sec. 4, Roosevelt Road, Taipei 10617, Taiwan, ROC
}

Available online 23 March 2006

\begin{abstract}
Cyclic loading during service may induce sub-surface defects in polymeric composite materials. These insidious defects may grow and eventually lead to catastrophic failures. The current work aims at exploring the possibility of using embedded fiber Bragg grating to monitor damage evolution. Fatigue damage evolution in Graphite/Epoxy coupons having a $[-45 / 90 /+45 / 0]_{2 s}$ lay-up sequence with a central circular hole subjected to cyclic loading is evaluated. This study suggested that the embedded fiber bragg gratings have good potentials to detect the appearance of damage. It is hoped that this methodology can be further developed as an online condition monitoring technique for safety-critical structures, thereby improving the safety and reliability of composite structures.
\end{abstract}

(C) 2006 Elsevier Ltd. All rights reserved.

Keywords: Delamination; Fatigue damage; Gr/Epoxy composite; Fiber optic sensor; Fiber bragg gratings

\section{Introduction}

Polymeric composite materials have better specific stiffness/strength, corrosion resistance, and most importantly, their directional properties may be tailor made. They are however prone to develop internal damage caused by tool drop, bird-strike, hail-storm and cyclic loading. Fatigue failure in composites is primarily due to one or more of the following: delamination, debonding, matrix cracking and fiber breakage [1].

Recently, there are general interests in the development of smart sensors integrated into structures to ensure continuous structural integrity monitoring [2-4]. A fiber bragg grating (FBG) is one of such element that finds increasing applications as a sensor in aerospace, structural, medical and chemical applications for vibration, temperature, strain, impact and general structural health monitoring [5-12]. These sensors are light, have small size, good sensitivity, good long-term stability, corrosion resistance and are immune to magnetic and electromagnetic interferences. Their small sizes and compatibility with common poly-

\footnotetext{
* Corresponding author.Tel.: +8862 3621 522; fax: +8862 3631755 .

E-mail address: csshin@ntu.edu.tw (C.S. Shin).
}

meric materials make them easily embeddable inside a structure without inducing significant weakening of the material. Several reports [13-17] have shown that on-line monitoring of the emergence and development of these defects is one of the key technologies for smart materials, thus permitting us to improve the safety and reliability of built-up composite structures.

This paper examines the responses of embedded FBG sensors in composites under cyclic loading.

\section{Experimental procedures}

The FBGs were fabricated in a $\mathrm{Ge}-\mathrm{B}$ co-doped single mode fiber by side writing using a phase mask with a period of $1.05 \mu \mathrm{m}$. The sensing length of the FBGs were $10 \mathrm{~mm}$. The reflectivity of the resulting FBG was about $99 \%$, and the peak wavelengths were from 1540 to $1550 \mathrm{~nm}$. The FWHM (full width half maximum) of the FBGs was about $0.175 \mathrm{~nm}$. Up to four FBGs with different reflected wavelengths were connected up in the same train to facilitate data logging. Reflected spectra from the FBGs were measured by an Anritsu optical spectrum analyzer (MS9710C OSA). Measurements were made when the specimen was free and again under a $10 \mathrm{kN}$ load after 
completed a certain amount of load cycling. The onset of fatigue damage was assessed through the changes in waveform shape and shift in wavelength for individual fiber grating sensors as well as through the secondary peaks in the optical spectrum waveform.

Fatigue tests were carried out on a 16 layer quasi-isotropic T300/3501 carbon fiber epoxy laminate specimen with a lay-up of $[-45 / 90 / 45 / 0]_{2 s}$. The test coupons were $30 \mathrm{~mm}$ wide and $2 \mathrm{~mm}$ thick, with a $6.3 \mathrm{~mm}$ diameter center hole. The fiber sensors were placed in between the central $0^{\circ}$ laminae along the axial loading direction during prepreg layup. The first sensor (designated FBG1) was embedded $\approx 1.5 \mathrm{~mm}$ from the hole tip. Subsequent sensors were located at an interval of $1.5 \mathrm{~mm}$. A strain gage was also stuck on the surface of the composite specimen just besides the central hole for specimen stiffness monitoring. The specimen was cyclically loaded by an MTS servo-hydraulic test system between 1.5 and $15 \mathrm{kN}$ at a frequency of $4 \mathrm{~Hz}$.

\section{Basic properties of fiber bragg grating sensors}

When a broadband light is coupled into an optical fiber with a uniform Bragg grating, a single peak with wavelength $\lambda$ satisfying the following relation will be reflected while the other wavelengths will be allowed through:

$\lambda=2 n_{\mathrm{e}} \Lambda$

where $n_{\mathrm{e}}$ is the effective refractive index and $\Lambda$ is the periodicity of the grating. If the uniformity of the grating period is perturbed, the single peak reflected spectrum will become broadened or chirped. When either or both of the $n_{\mathrm{e}}$ and $\Lambda$ change, the center wavelength of the reflected spectrum shifts. $\Lambda$ will be changed if the FBG is subjected to a deformation. Such deformation may be caused by mechanical or thermal strains. $n_{\mathrm{e}}$ will be affected by variation in temperature and the three-dimensional stress state acting on the fiber. In general, the reflected wavelength will shift by $\sim 1 \mathrm{pm}$ under a strain of $1 \mu \varepsilon$ or a temperature change of $0.1^{\circ} \mathrm{C}$. If temperature variation is negligible, then the change in the spectrum basically reflects a change in the stress/strain status on the FBG. A preliminary cyclic test on the current specimens showed that a maximum steady state temperature rise of $5{ }^{\circ} \mathrm{C}$ will occur at the tip of the hole when loaded at $10 \mathrm{~Hz}$ when the specimen is enclosed in a foam chamber. Temperature change is negligible $5 \mathrm{~min}$ after cyclic loading was stopped when test was carried out in ambient laboratory condition. Hence, all spectrum measurements were made at least five minutes after cyclic loading stopped.

\section{Results and discussion}

Fig. 1 shows the development in laminate stiffness versus cycle as monitored by a strain gage stuck next to the edge of the hole. From 0 to 10,000 cycles, the laminate stiffness showed a slight and gradual increase. It started to show a slight drop between the 10,000 and 20,000 cycles. At

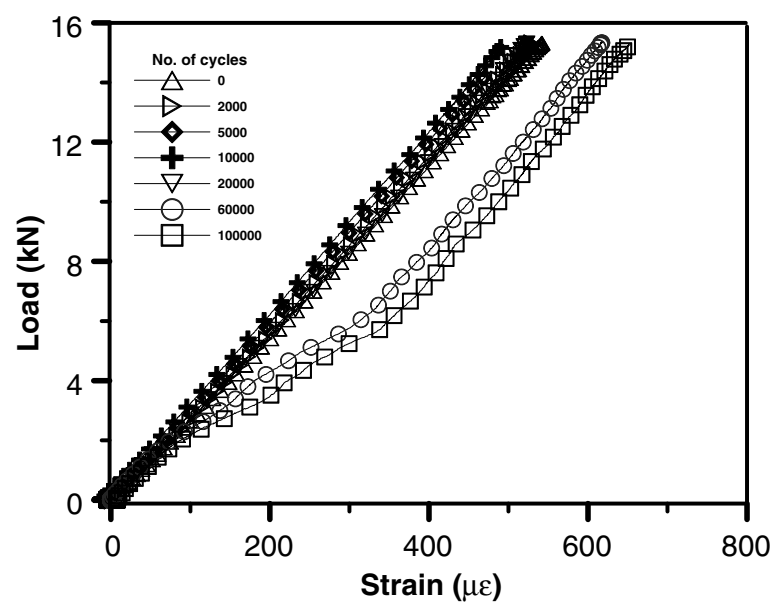

Fig. 1. Specimen stiffness variation with loading cycles.

60,000 cycles, significant departure from linearity and drop in stiffness was recorded. The stiffness continued to drop until the test was interrupted at 100,000 cycles. The nonlinearity in the load-deformation traces is associated with matrix cracking, which is evident in the following figures. The specimen was sectioned according to the schematic cutting plan shown in Fig. 2 and examined under an optical microscope.

Fig. 2a-d shows the sectional views of the laminate progressively further away from the hole edge as indicated in the schematic cutting plan. These sections are in the vicinity of the embedded fiber gratings FBG1 through FBG4. Matrix cracking occurred extensively in Fig. 2a which is the nearest to the edge of the hole. Some of these cracks have been confined in the $90^{\circ}$ laminae (denoted by $\mathrm{M}$ in the figure) while some have extended into the neighboring $\pm 45^{\circ}$ laminae (denoted by $\mathrm{T}$ ). In the latter cases, delamination (D) often occurred connecting the matrix cracks in different laminae. At $3 \mathrm{~mm}$ from the hole edge, matrix cracking can also been seen (Fig. 2b) but is not so extensive and are mostly confined to the $90^{\circ}$ laminae. Matrix cracking occurs more sparingly as we go further from the hole (see Fig. 2c and d). Fig. 2e shows a broader view of the section on the same plane as Fig. 2a. Matrix cracking is mainly seen in the stress concentration region near the hole. Fig. $2 \mathrm{f}$ shows the section through the hole in a direction perpendicular to the loading direction. Damage is not evident when viewing in this direction. Cross sectional views of the specimen show that FBGs merged well with the surrounding graphite fibers without inducing a defective matrix-rich region around them. Crack densities at different locations are estimated from these micrographs and are shown in Fig. 3.

Fig. 4 compares the ultrasonic $\mathrm{C}$-scan images at 0 , $10,000,60,000$ and 100,000 cycles. The irregularities on the left half of the $\mathrm{C}$-scan image are due to strain gage and its accessories. The FBGs were embedded on the right half. Since the matrix cracks are lying in planes in the same direction as the ultrasonic wave propagation, 


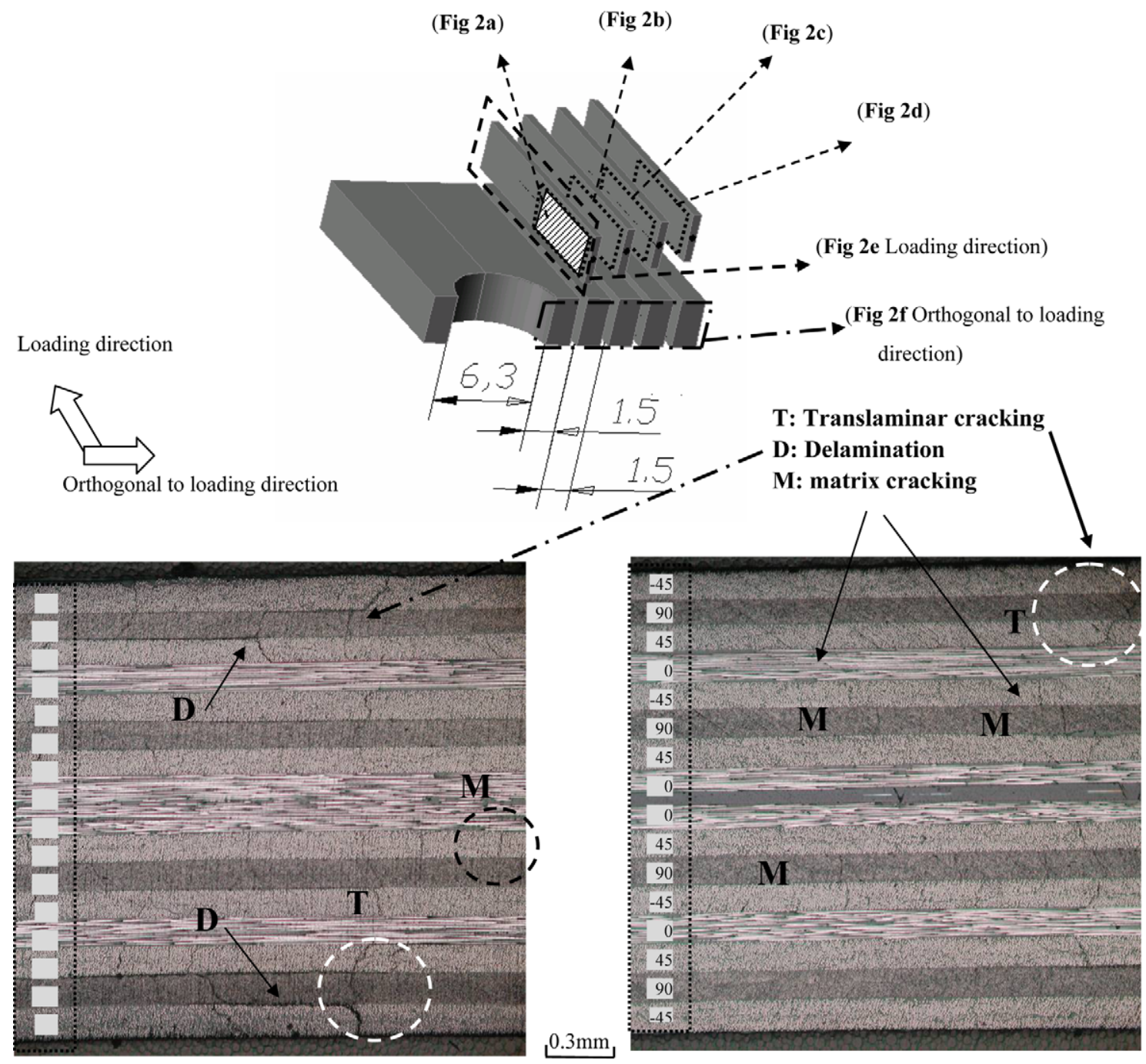

(a)

(b)

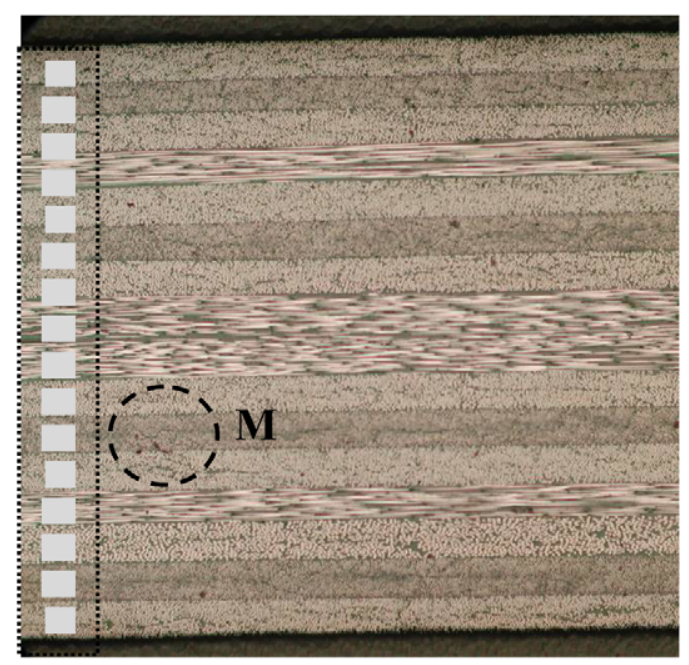

(c)

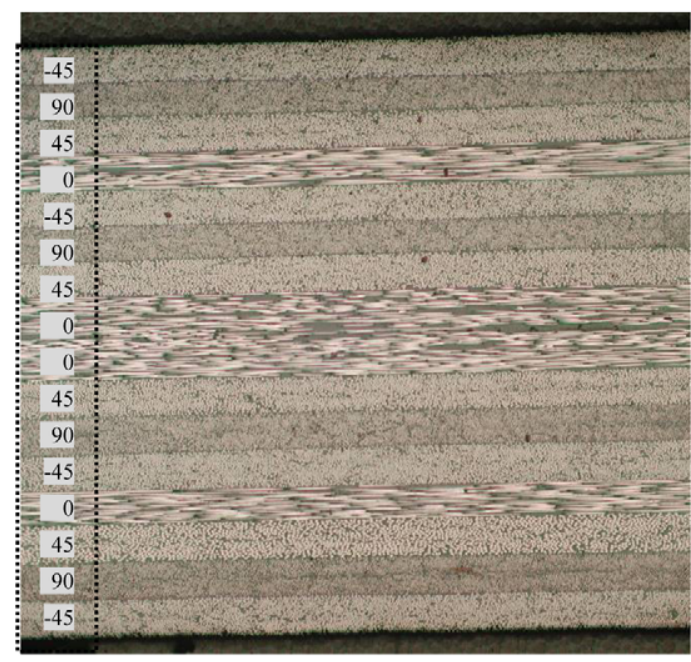

(d)

Fig. 2. Optical micrographs on the sectional view of the fatigued specimen.

they will not be detected by the C-scan. However, the occurrence of delamination at the later stage of matrix crack development will be picked up by the C-scan.
Fig. 4b shows that at 10,000 cycles, the damage zone has reached the FBG1 position. By 60,000 and 100,000 cycles, the damage zone is approaching FBG2. This 


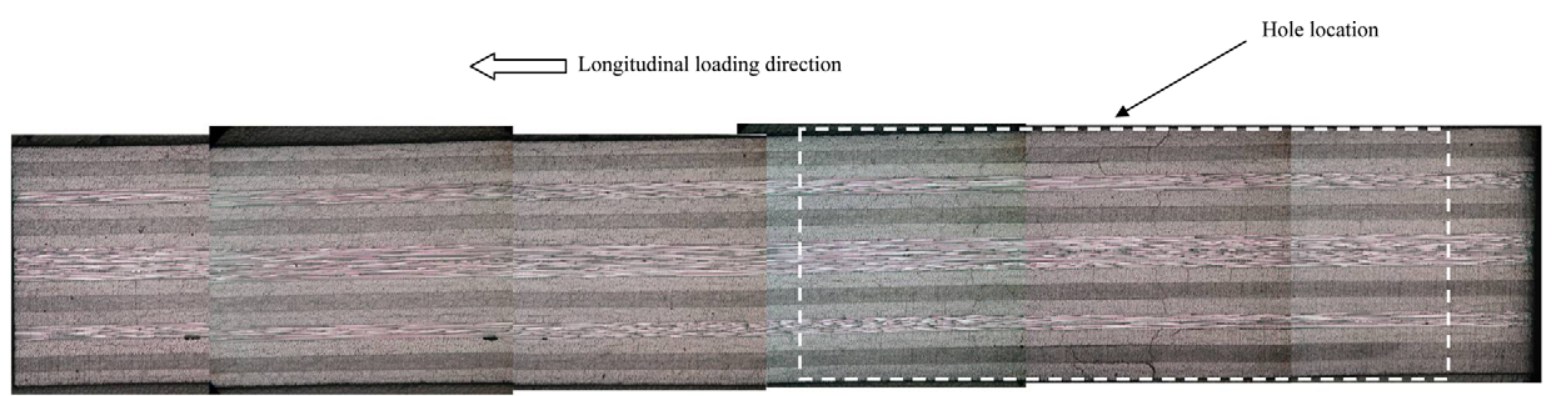

(e)

$0.7 \mathrm{~mm}$

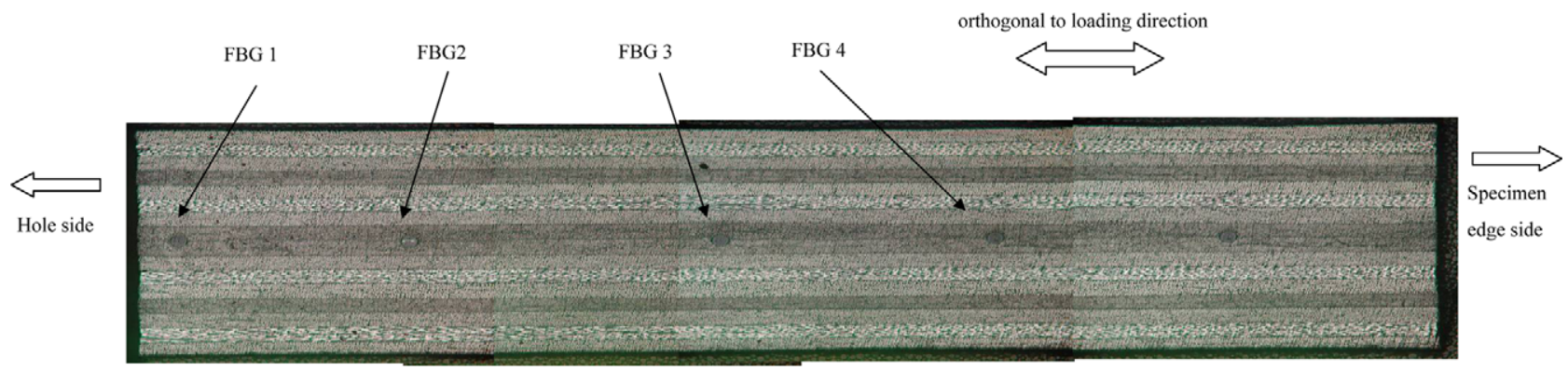

(f)

$0.7 \mathrm{~mm}$

Fig. 2 (continued)

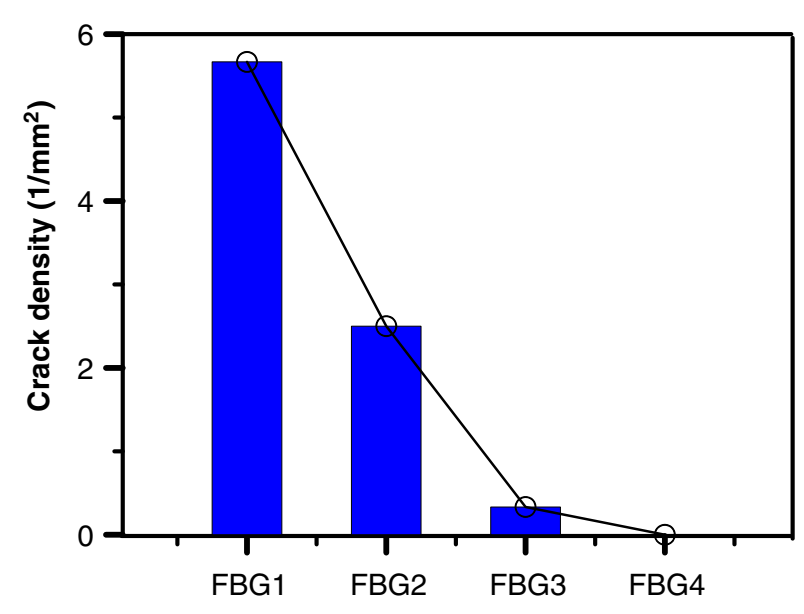

Fig. 3. Crack density distribution in the vicinity of the embedded FBGs.

suggests that preliminary matrix cracking probably exists at the FBG2 position but it has not yet developed into the delamination stage.

Comparison of the FBG spectra just before embedding and after curing and hole drilling in the composite laminate shows that a tensile residual strain occurred in FBG1 and FBG3 locations while FBG2 was under a compressive residual strain. FBG4 was probably broken during the curing process as no signal was obtained thereafter. Fig. 5 shows the spectra under load free and $10 \mathrm{kN}$ tension conditions after fatiguing for different number of cycles up to 100,000 cycles. Under the load free condition (Fig. 5a-c), the peaks of FBG1 through FBG3 more or less maintained their overall shapes while the centre wavelengths have shifted and the intensities changed as loading cycle increased. Fig. 6 shows the amount of wavelength shifts in the three FBGs under the load free condition. A rapid increase in wavelengths occurs initially before 5000 cycles. Wavelength shifts reached a peak at about 30,000 cycles. Thereafter, they started to decrease gradually. Also shown in Fig. 6 is the result corresponding to FBG1 obtained from another specimen. It suggests the wavelength shift behavior is quite reproducible. This shift towards longer wavelengths may be accompanied by a dynamic creep of the laminate as it was tested under a tension-tension cycling under load control. Strain monitoring during tension-tension cyclic loading in other work [18] also showed an initial sharp rise in strain followed by a more gradual increase. Since all three FBGs shows the same behavior in wavelength shift, the abrupt change in the rates of increase in wavelength shift at 5000 cycles is probably not related to internal damages which should vary in extent at different positions.

Under a $10 \mathrm{kN}$ load, the spectrum of the three FBGs behaved quite differently (see Fig. 5d-f). For FBG1, significant chirping occurred even before the cyclic loading, presumably due to the strain gradient along the FBG induced by the hole. As fatigue cycling increased, the lower and upper limits of the chirped spectrum shifted by different extent and sometimes to different directions. Fig. 7 shows the incremental shifts in these limits with respect to the wavelength before cyclic loading. Before 10,000 cycles, the limits both shift in the positive direction. This probably reflects the same tensile plastic deformation that led to initial abrupt wavelength shift under zero load mentioned above for Fig. 6. Beyond 10,000 cycles, the upper limit continues to shift in a more gentle manner towards the positive 


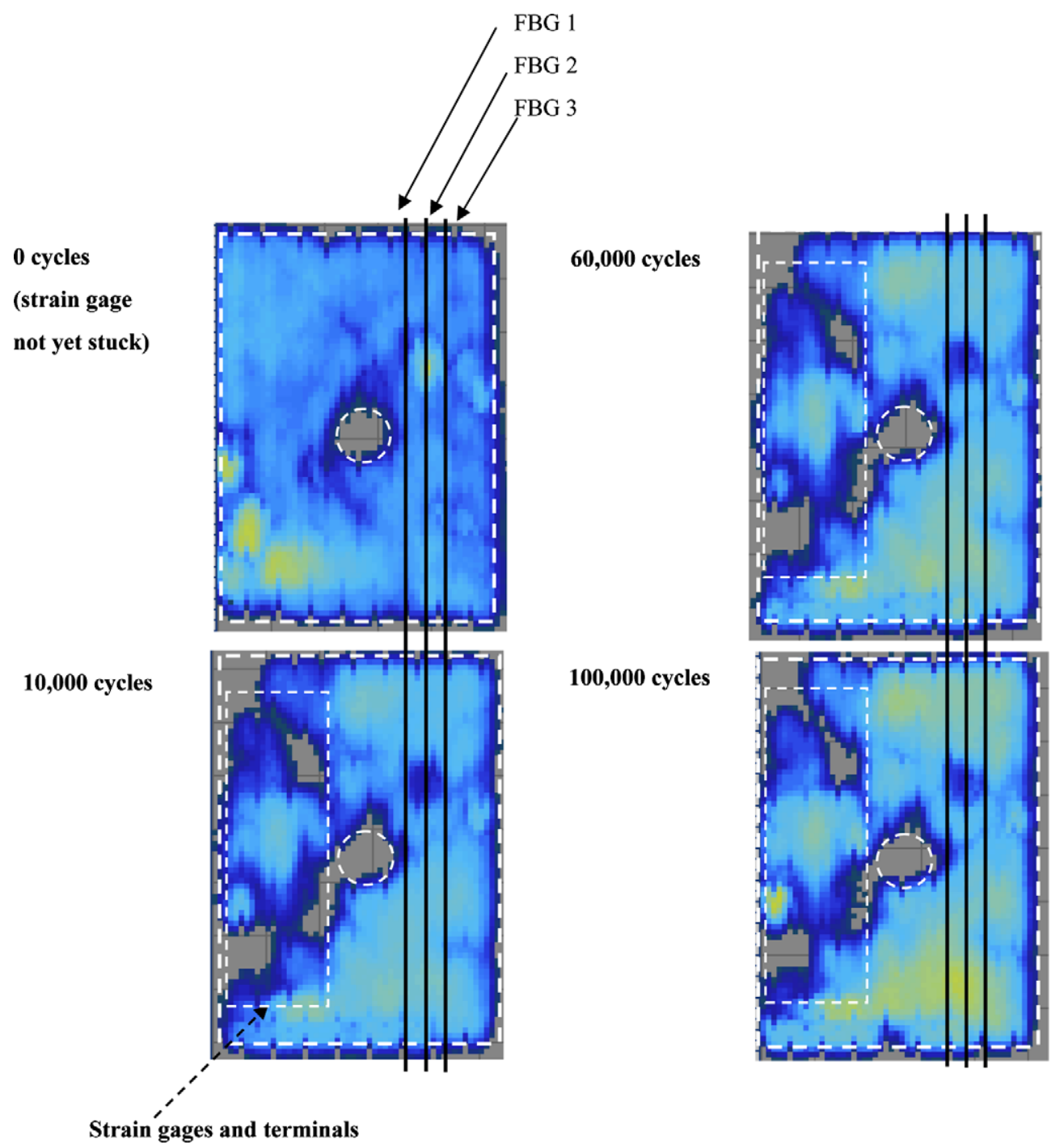

Fig. 4. C-scan patterns under different loading cycles.

direction while the lower limit to the negative direction. This phenomenon probably reflected the occurrence of fatigue damage that involved occasional matrix cracking and localized delamination. Under the applied load, localized strain on the FBG ahead of the damages will be intensified, leading to an upper spectrum limit shift to the right. On the other hand, matrix strain in the vicinity of the cracked region will be released, leading to a lower strain than the crack-free condition and a shift of the lower spectrum limit to the left will occur. Such a differential shift on the upper and lower limits leads to an increase in the width of the chirped spectrum.

The chirping was also observed in FBG2. However, due to unknown reason, the reflectance quality of FBG2 degraded to only slightly above the baseline noise level under load. This makes further analyses difficult.

For FBG3, a well-defined peak with similar intensity occurred throughout the loading history, suggesting that matrix cracking has not developed yet. The center wavelength of the peak has shifted to the right as loading cycle increased (see Fig. 7). The amount of shift was larger in the beginning but the increase in shift slowed down beyond 20,000 cycles. It is thought that this pattern of shift is the same as that described above for Fig. 6.

Besides wavelength shift, the FBG spectra shown in Fig. 5 under $10 \mathrm{kN}$ loading revealed a change in intensities as well. Fig. 8 compares the change in intensity referring to virgin state for the three FBGs after different loading cycles. For FBG1, a sharp drop in intensity occurred between 10,000 and 20,000 cycles. This period corresponds to the start of differential shift in the upper and lower limits of the chirped spectrum (Fig. 7) and may have something to do with appearance of matrix cracking. Thereafter, it increased gradually. For FBG2, gradual increase occurred until 60,000 cycles, after which intensity started to drop. In the vicinity of FBG2, microscopic sectioning only revealed occasional matrix cracking. C-scan examination (Fig. 4) also indicated delamination has not yet spread to this location. The intensity drop may therefore be associated with the appearance matrix cracking as well as the perturbation 

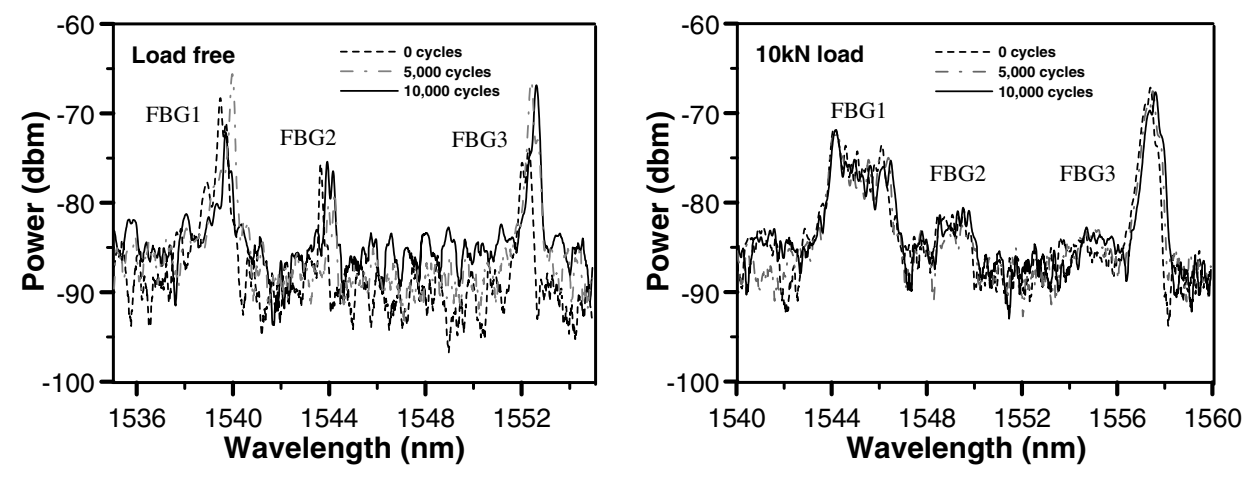

a

$0,5,000$ and 10,000 cycles

d

$0,5,000$ and 10,000 cycles
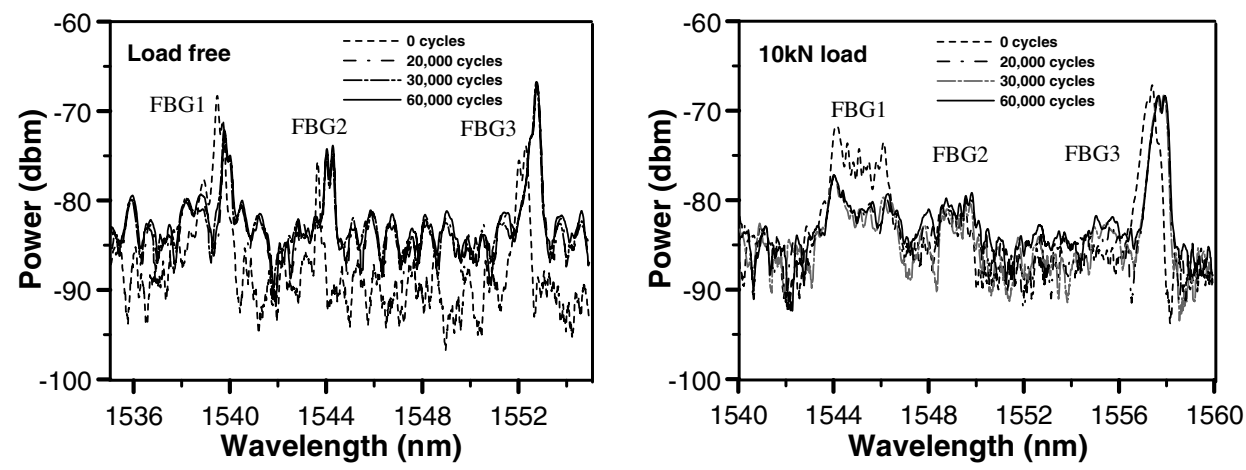

b

$20,000,30,000$ and 60000 cycles

e

$20,000,30,000$ and 60000 cycles
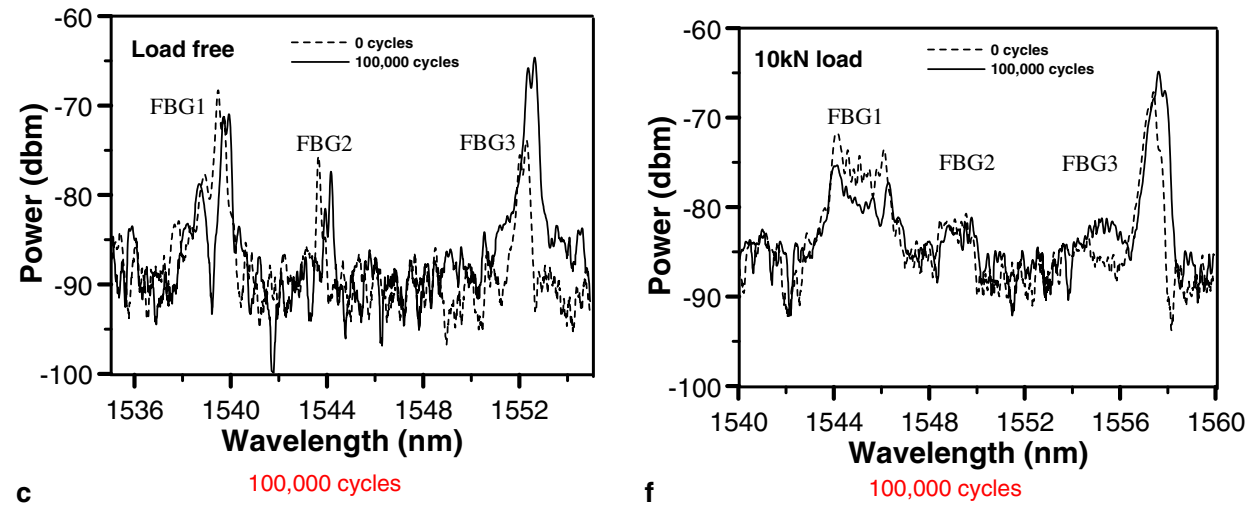

Fig. 5. FBG Spectra after different loading cycles under (a-c) zero load and (d-f) $10 \mathrm{kN}$.

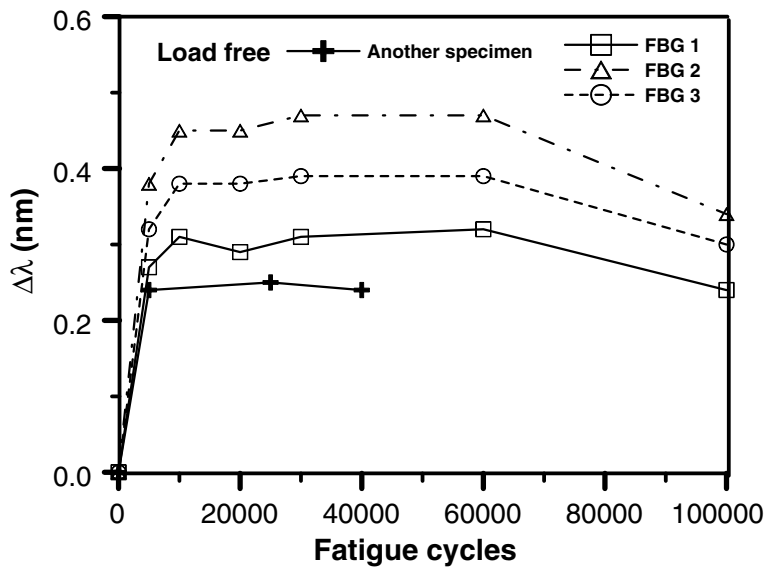

Fig. 6. Wavelength shift of FBGs under load free condition.

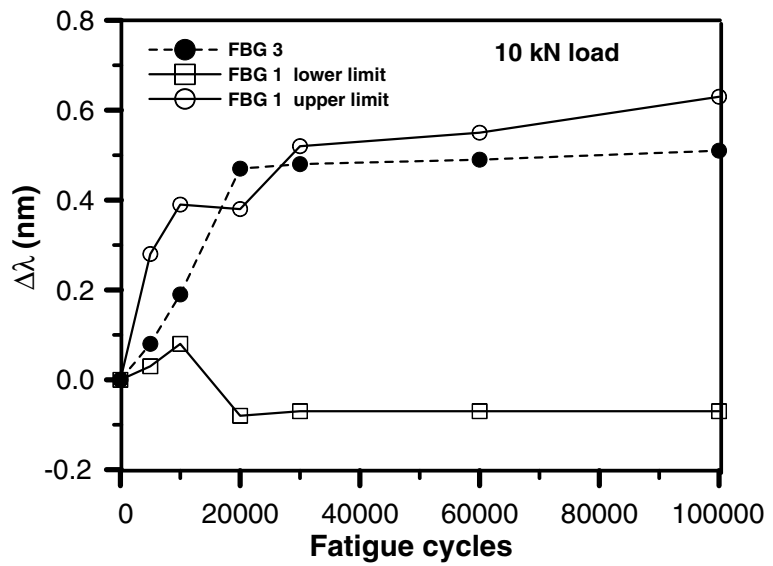

Fig. 7. Wavelength shift of FBGs under $10 \mathrm{kN}$ load. 


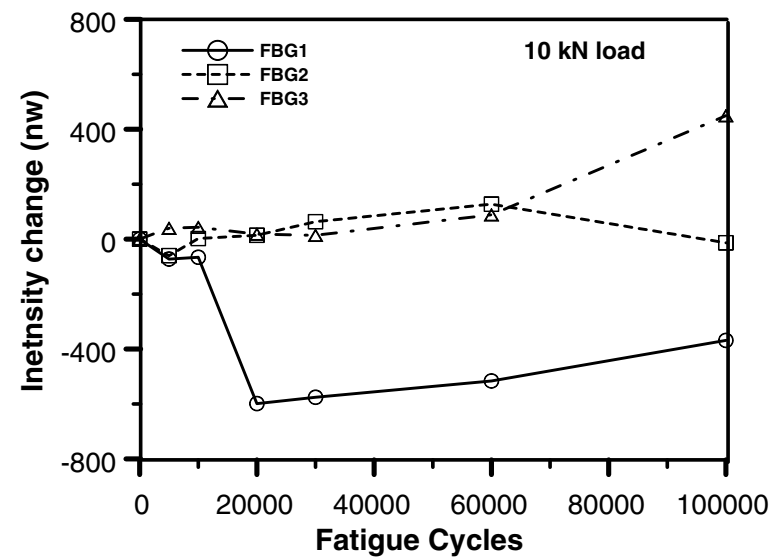

Fig. 8. Intensity changes of the FBG spectra versus loading cycles.

of the residual stress field due to intensification of damage near the hole tip. For FBG3, the intensity was increasing with loading cycles. Incidentally, $\mathrm{C}$-scan results showed the damage zone was still far away from FBG3. More tests are needed to confirm that abrupt intensity drop is related to the development of matrix cracking.

In the current work, the FBGs were embedded between two $0^{\circ}$ laminae and so were not exposed to direct contact with matrix cracks and delamination. It is postulated more information may be gained if the FBGs are embedded between $0^{\circ}$ and $90^{\circ}$ laminae or between $0^{\circ}$ and $45^{\circ}$ laminae.

\section{Conclusions}

The reflected spectrum of an embedded FBG sensor will shift and deform when fatigue damage occurs. The associated broadening of the peak and the sharp drop in light intensity seems to be associated with the onset of matrix cracking and localized delamination. The shift in the wavelength reflects the change in the internal strain around the FBG but no particular association with fatigue damage is observed.

\section{Acknowledgements}

This work was carried out with support of the National Science Council projects (NSC91-2212-E-002-056 and NSC92-2212-E-002-002). We are also indebted to Professor S. K. Liaw of NTUST for some of the equipment support and advice.

\section{References}

[1] Talreja Ramesh. Fatigue of composite materials. Lancater. Penn. USA: Technomic Publishing Co., Inc.; 1987. 3-58.

[2] Nobuo Takeda, Tadahito Mizutani, Kentaro Hayashi, Yoji Okabe. Application of fiber bragg grating sensors to real-time strain measurement of cryogenic tanks. Proc SPIE 2003;5056:304-11.

[3] Skontorp A. Structural integrity of quasi-isotropic composite laminates with embedded optical fibers. J Reinf Plast Compos 2000;19(13):1056-77.

[4] Hadzic R, John S, Herszberg I. Structural integrity analysis of embedded optical fibres in composite structures. Compos Struct 1999;47(1):759-65.

[5] Yoji Okabe, Shigeki Yashiro, Tatsuro Kosaka, Nobuo Takeda. Detection of transverse cracks in CFRP composites using embedded fiber bragg grating sensors. Smart Mater Struct 2000;9(6):832-8.

[6] Rao YJ. Recent process in applications of in-fibre bragg grating sensors. Opt Lasers Eng 1999;31:297-324.

[7] Tomasel FG, Laura PAA. Assessing the healing of mechanical structures through changes in their vibrational characteristics as detected by fiber optic bragg gratings. J Sound Vib 2002;253:523-7.

[8] Hill Kenneth O, Meltz Gerald. Fiber bragg grating technology fundamentals and overview. J Lightwave Technol 1997;15:1263-76.

[9] Leng JS, Asundi A. Non-destructive evaluation of smart materials by using extrinsic Fabry-Perèt interferometric and fiber bragg grating sensors. NDT \& E Int 2002;35:273-6.

[10] El-Sherif MA, Radhakrishnan J. Advanced composites with embedded fiber optic sensors for smart applications. J Reinf Plast Compos 1997;16:144-54.

[11] Lau Kin-tak, Yuan Libo, Zhou Li-min, Wu Jingshen. Chung-ho Woo Strain monitoring in FRP laminates and concrete beams using FBG sensors. Compos Struct 2000;51:9-20.

[12] Leng J, Asundi A. Structural health monitoring of smart composite materials by using EFPI and FBG sensors. Sensor Actuator A 2003; 103:330-40.

[13] Yoji Okabe, Ryohei Tsuji, Nobuo Takeda. Application of chirped fiber bragg grating sensors for identification of crack locations in composites. Compos Part A: Appl Sci Manufact 2004;35(1):59-66.

[14] Shinji Ogihara, Nobuo Takeda, Satoshi Kobayashi, Akira Kobayashi. Damage mechanics characterization of transverse cracking behavior in quasi-isotropic CFRP laminates with interlaminartoughened layers. Int J Fatigue 2002;24(2-4):93-8.

[15] Yoji Okabe, Tadahito Mizutani, Shigeki Yashiro, Nobuo Takeda. Detection of microscopic damages in composite laminates with embedded small-diameter fiber bragg grating sensors. Compos Sci Technol 2002;62(7-8):951-8.

[16] Nobuo Takeda. Characterization of microscopic damage in composite laminates and real-time monitoring by embedded optical fiber sensors. Int J Fatigue 2002;24(2-4):281-9.

[17] Yoji Okabe, Nobuhira Tanaka, Nobuo Takeda. Effect of fiber coating on crack detection in carbon fiber reinforced plastic composites using fiber bragg grating sensors. Smart Mater Struct 2002;11(6):892-8.

[18] Seo Dae-Cheol, Lee Jung-Ju. Damage detection of CFRP laminates using electrical resistance measurement and neural network. Compos Struct 1999;47(1):525-30. 